\title{
The Brightness and Polarization Structure of Compact Radio Sources
}

\author{
Raymond Rusk \\ Dept. of Astronomy \\ Univ. of Toronto \\ Toronto, Ontario, M5S 1 A1
}

New VLA polarization data for 140 radio sources have been used to study the correlation between VLBI structural position angle and radio and optical polarization position angles reported in Rusk and Seaquist (1985). We confirm that there is a strong tendency, in active galaxies and quasars, for the radio $\mathbf{E}$ vector (of the core component) to lie normal to the VLBI structural axis. However, in BL Lac objects we find a tendency for the core radio $\mathbf{E}$ vector to be aligned parallel to the VLBI structural axis.

\section{THE SURVEY}

Many compact sources when observed at VLBI resolution display linear asymmetric structures consisting of an (apparently) optically thick core and an (apparently) optically thin jet. If the low-frequency turnover seen in the spectra of these sources is due to synchrotron self-absorption, then the optically thick core component should have a low degree of polarization. Most of the observed polarization must arise in the optically thin jet component which suggests that the direction of the radio $\mathbf{E}$ vector will be correlated with the direction of the VLBI asymmetry.

From a comprehensive literature search (complete to January 1985) we created a list of 156 sources, including 3 sources which we had mapped in a VLBI survey of highly polarized sources, which had VLBI structural position angles given by either hybrid maps or models. VLA snap-shots for 140 of these sources were made (at 2, 6, 18 and $22 \mathrm{cms}$ ) and VLA polarization measurements for an additional 14 sources were taken from the literature.

All of our data were self-calibrated, mapped and CLEANED using the NRAO AIPS package. Dynamic ranges of several-thousand-to-one were obtained in most cases. Offcore emission was mapped out so that core polarization values would reflect polarization from sub-arcsecond structures. For extended sources observed in both A- and B-arrays, scaled-array observations were used to form rotation measure and spectral index maps. The polarization data were corrected for positive bias, and the integrated rotation measure (RMc) and intrinsic polarization angle (IPAc) values for the core components were estimated.

\section{DISCUSSION OF RESULTS}

The left half of Figure 1 shows the misalignment angle distribution between VLBI structural and radio polarization position angles for 102 active galaxies and quasars. There is a sharp peak near $90^{\circ}$ indicating that the sub-arcsecond magnetic field structure is aligned with the VLBI axis in most sources. The probable error in the misalignment value for any given source is roughly the bin size, so much of the scatter away from $90^{\circ}$ is accounted for by error. However, some sources show large curvatures in their milliarcsecond jets or 
have continuously curved milliarcsecond to arcsecond scale structures. It is to be expected then that some large misalignments are real.

In light of the above discussion, it appears that for the great majority of sources, either their sub-arcsecond jets are fairly straight in the region producing the polarized emission and align with the observed milliarcsecond structure, or the observed polarization comes from a region near the observed VLBI jets. For about $30 \%$ of the sources, no arcsecond scale structure was found in the maps and the core was unresolved at subarcsecond resolution. For these sources at least, it is likely that nearly all the polarized flux comes from the observed VLBI structures.

The right half of Figure 1 shows the misalignment angle distribution between core radio polarization and VLBI position angles for $22 \mathrm{BL}$ Lac objects. The slight bimodality apparent in the active galaxy and quasar distribution is very evident for the population of BL Lac objects. Some BL Lac objects have highly variable radio polarization making RMc and IPAc values uncertain (for these sources). However, variability alone is unlikely to produce this markedly bimodal distribution.

The tendency in BL Lac objects for the integrated polarization position angle to lie along the VLBI jet agrees with observations of BL Lacertae itself (Aller and Aller, 1984 and Mutel and Phillips, 1984) during times of outburst. (BL Lacertae has not been included in Figure 1.) Axial compression of the magnetic field in a shock could produce a magnetic field nearly perpendicular to the jet, as they discuss, and the strong outbursts of radio emission might result from electron enhancements within the shock.

While our analysis is still preliminary, and has not included a detailed study of the misalignment angle distribution for individual VLBI morphological classes, we can conclude that in most quasars and active galaxies there is a preferred structural axis defined by the VLBI morphology and that the sub-arcsecond magnetic field runs along this axis. The misalignment angle distribution for BL Lac objects is apparently distinct from that of quasars, in that the radio $\mathbf{E}$ vector is frequently aligned parallel, not normal, to the VLBI axis in these objects.
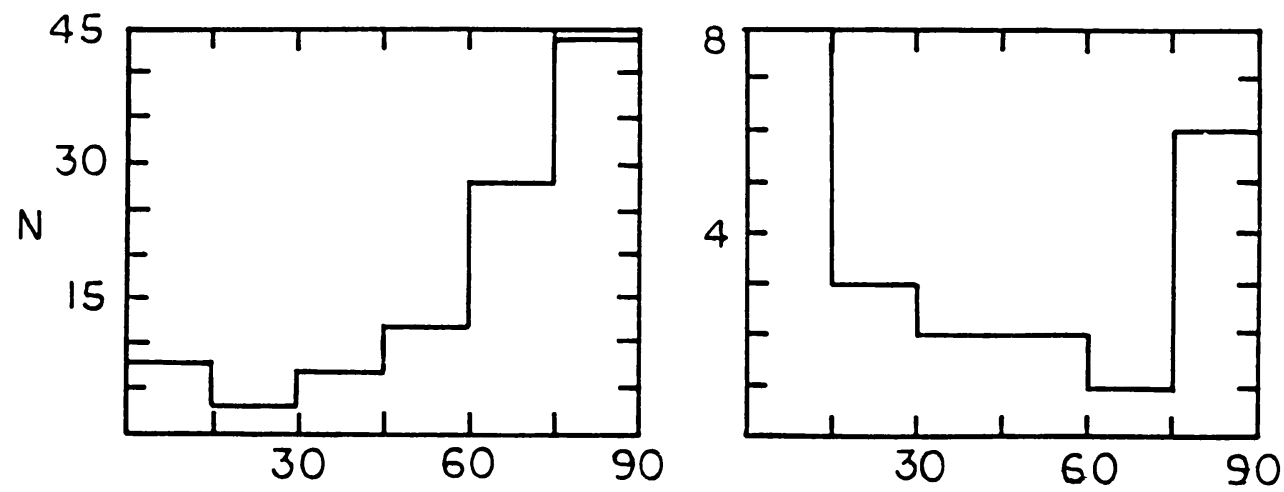

\section{MISALIGNMENT (DEG)}

Fig. 1. The distribution of the acute angle between VLBI structural axis and intrinsic (core) radio polarization $\mathbf{E}$ vector for active galaxies and quasars (Left), and BL Lac objects (Right).

\section{REFERENCES}

Aller, H.D., and Aller, M.F.: 1984, IAU Symp. 110, p. 119.

Mutel, R.L., and Phillips, R.B.: 1984, IAU Symp. 110, p. 117.

Rusk, R. and Seaquist, E.R.: 1985, Astron. J. 90, 30. 